\title{
SISTEMA DE INSTRUMENTAÇÃO WIRELESS EM FORNOS ROTATIVOS*
}

Everton Azola de Mattos ${ }^{1}$

\section{Resumo}

$\mathrm{Na}$ operação da Anglo American localizada em Barro Alto, utiliza-se 2 (dois) Fornos Rotativos no processo de fabricação de ferroníquel. Esses fornos possuem 6,2m de diâmetro e $185 \mathrm{~m}$ de comprimento, no qual é necessário o monitoramento de temperatura (20) e vazão (4) ao longo do forno. Originalmente essas medições eram realizadas através de 2 PLC's embarcados nos fornos, no qual recebiam as medições via cabos em bandejamentos. Esses PLC's se comunicavam via rádio modem com os PLC's principais dos Fornos. Durante a operação encontramos diversas dificuldades de manutenção e confiabilidade, já que os fornos operam em regime de $24 \mathrm{~h}$ por dia e 7 dias por semana. Por várias vezes alguns cabos se danificavam devida a alta temperatura dos fornos, as bandejas que se desprendiam, falhas de cartões dos PLC's, etc. Com isso as medições eram interrompidas. Para solucionar esse problema, foi desenvolvido um sistema de Instrumentação Wireless. Esse sistema basicamente é a instalação de instrumentos com a tecnologia WirelessHart no qual os instrumentos não possuem alimentação por cabos e sim por baterias e a comunicação é feita via sinal wireless. Esses instrumentos apresentam alta confiabilidade nas medições no qual foi possível retirar toda a infraestrutura antiga e garantir continuidade/confiabilidade nas medições.

Palavras-chave: Wireless; Wirelesshart; Instrumentação.

\section{WIRELESS INSTRUMENTATION IN ROTARY KILNS}

\begin{abstract}
At the Anglo American plant located in Barro Alto, two (2) Rotary Kilns are used in the iron-nickel manufacturing process. These furnaces are $6,2 \mathrm{~m}$ in diameter and $185 \mathrm{~m}$ in length, in which it is necessary to monitor temperature (20) and flow (4) along the kiln. Originally these measurements were carried out through 2 PLCs that were shipped in the kilns (rotated with the kiln), in which they received the information of the Instruments through cables allocated in the trays. These PLCs communicated via wireless radio modem with the main PLCs of the Kilns. During the operation we encounter several difficulties of maintenance and reliability, the Kilns operates on a $24 \mathrm{~h}$ a day and 7 days a week basis. For several times some cables were damaged due to the high temperature of the furnaces, the trays that were dislodged, card failures of the PLC's, etc. This interrupted the measurements. To solve this problem, a Wireless Instrumentation system was developed. This system basically is the installation of instruments with WirelessHart technology in which the instruments do not have power by cables but by batteries and the communication is made via wireless signal. These instruments showed high reliability in the measurements in which it was possible to remove all the old infrastructure and to guarantee continuity / reliability in the measurements.
\end{abstract}

Keywords: Wireless, Wirelesshart, Instrumentation.

1 Engenharia Elétrica e Eletrônica, Pós-graduado, Coordenador de Instrumentação e Automação, Manutenção, Anglo American, Barro Alto, Goiás, Brasil. 


\section{INTRODUÇÃO}

\subsection{Fatores Motivadores}

Na planta da Anglo American localizada em Barro Alto, utiliza-se 2 (dois) Fornos Rotativos no processo de fabricação de ferro-níquel. Esses fornos possuem $6,2 \mathrm{~m}$ de diâmetro e $185 \mathrm{~m}$ de comprimento, no qual é necessário o monitoramento de temperatura (20 medições) e vazão (4 medições) ao longo do forno. No projeto original dos Fornos Rotativos a medição dos Instrumentos era executada por dois PLC's instalados no corpo do Calcinador (PLC onboard) que se comunicam com o PLC principal do forno através de um Ethernet Radio Modem. Os termopares e transmissores de vazão são conectados nesses dois PLC's através de cabos e bandejas fixadas no corpo do Calcinador. Esses cabos constantemente se queimavam devida a alta temperatura do forno, por diversas vezes tivemos problemas na fixação do bandejamento e alguns problemas no barramento de alimentação. Essas manutenções provocaram paradas que impactam diretamente a produção. A figura 1 abaixo mostra os fornos rotativos.

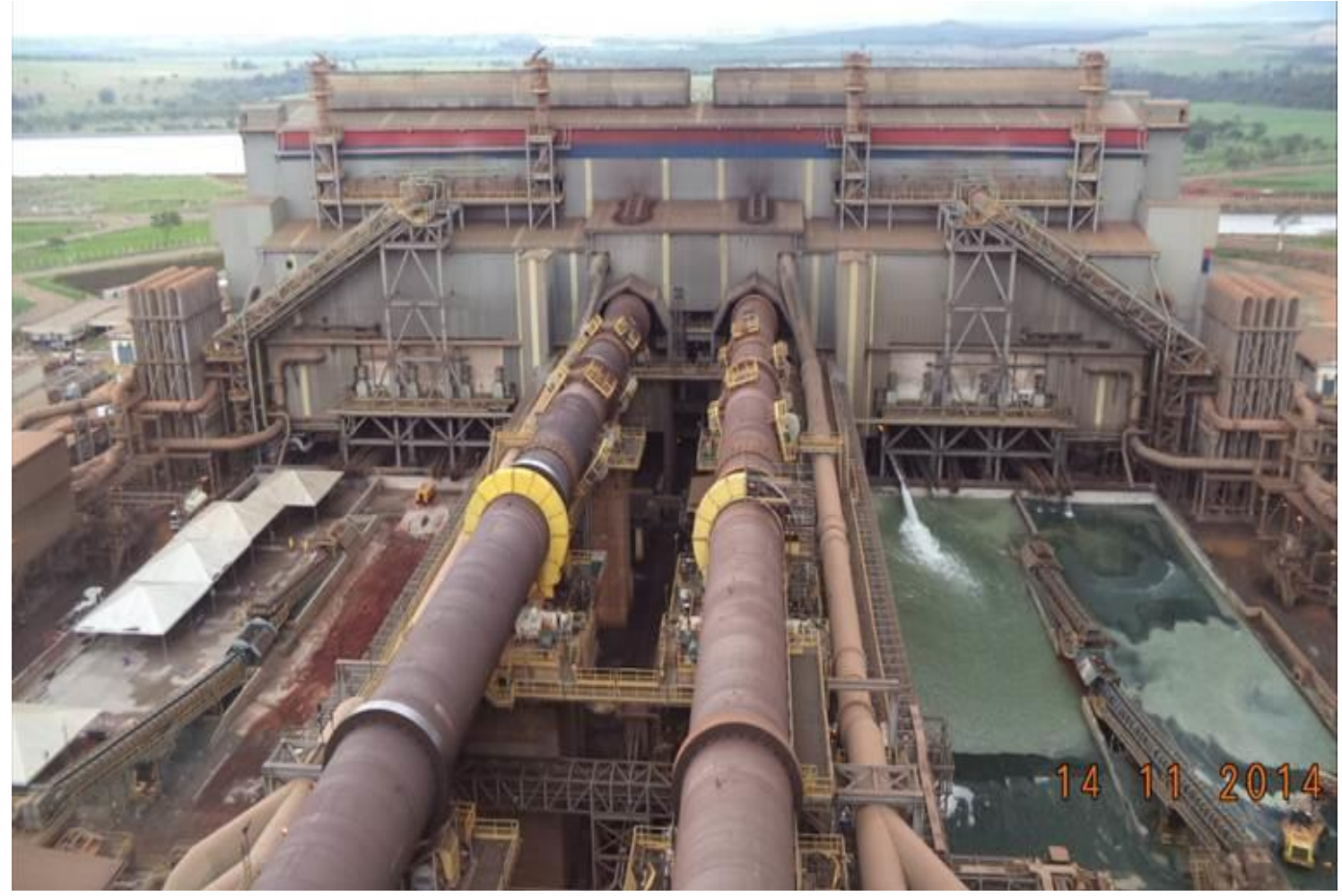

Figura 1 - Vista superior dos fornos rotativos 
Logo abaixo, a figura 2 mostra os pontos vulneráveis que o sistema anterior apresentava.

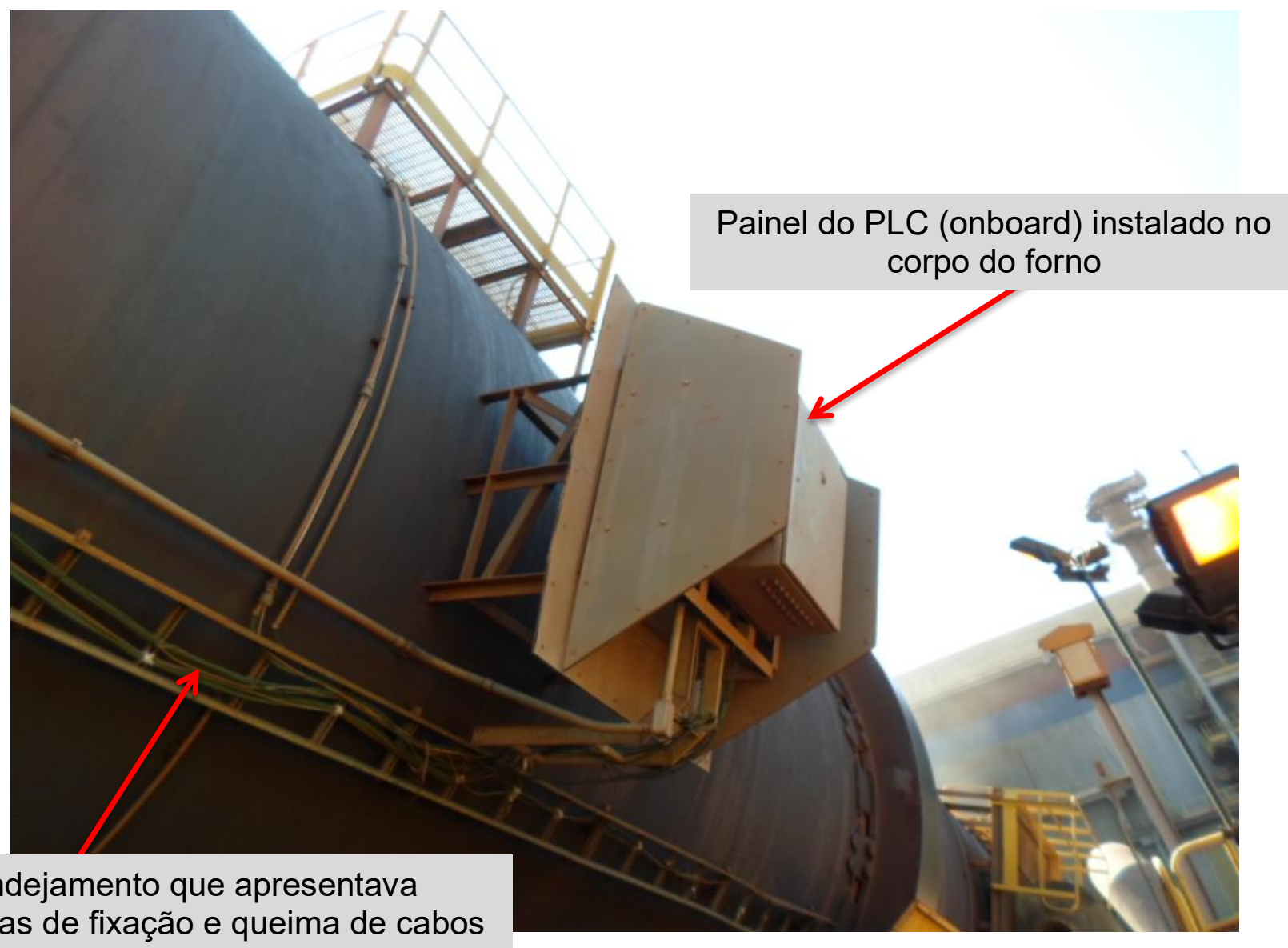

problemas de fixação e queima de cabos

Figura 2 - Painel on-board e bandejamento instalados no forno rotativo

\subsection{Objetivo}

Basicamente o objetivo desse projeto foi o desenvolvimento de um sistema de comunicação wireless para os 20 (vinte) instrumentos de temperatura e 4 (quatro) instrumentos de vazão instalados no corpo do Calcinador. Esse sistema eliminou os dois PLC's onboard, barramentos elétricos, cabos e bandejas, com o intuito de aumentar a confiabilidade dos fornos rotativos e qualidade do sinal transmitido.

\subsection{Desenvolvimento}

Para o desenvolvimento desse projeto foram estudadas diversas alternativas de transmissão de dados sem fio (wireless), mas deveria ser uma alternativa que apresentasse confiabilidade/integridade de transmissão e ainda fosse um sistema com capacidade de trabalhar em áreas agressivas (exposto a intempéries e a alta temperatura). 


\subsubsection{Escolha de tecnologia}

A tecnologia escolhida para esse projeto foi da fabricante EMERSON, no qual apresentou uma grande flexibilidade de Instrumentos que podem ser utilizados na rede WirelessHart. Outro ponto decisivo foi também de ser uma rede que suporta um maior número de instrumentos. A rede WirelessHart trabalha de forma que os próprios instrumentos além de enviar as suas medições eles também funcionam como repetidores da rede, aumentando consideravelmente a cobertura da rede. Na figura 3 mostra exemplos de Instrumentos que trabalham na tecnologia WirelessHart.

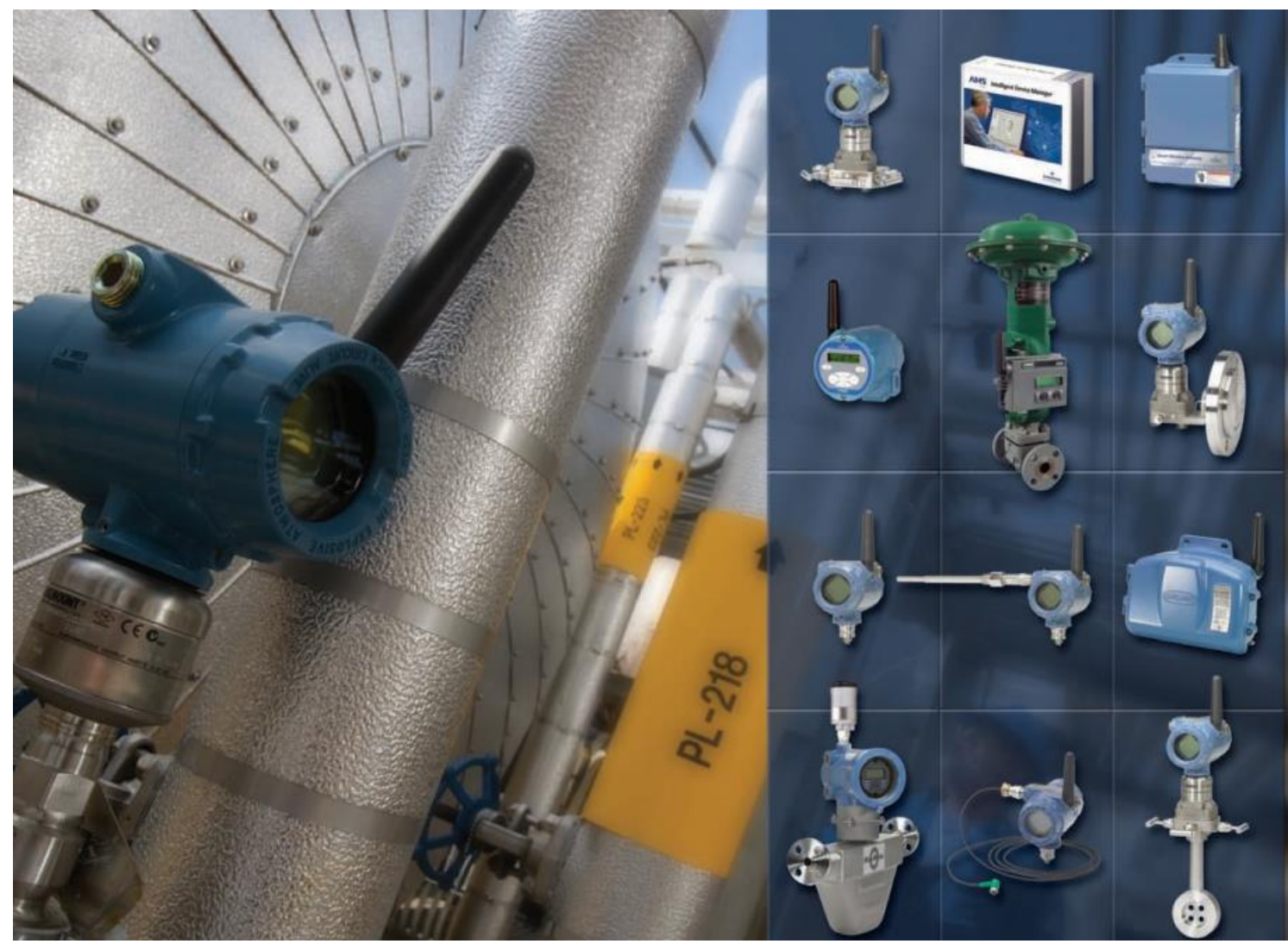

Figura 3 - Linha de produtos WirelessHart da Emerson

Outro ponto considerado nessa escolha de tecnologia foi a duração da bateria dos instrumentos, já que os mesmos deveriam funcionar por mais de um ano sem a necessidade de troca de bateria. Para isso foi utilizado um simulador da Emerson para verificar o tempo máximo que a bateria teria de vida útil. No projeto foi utilizada uma taxa de atualização de $8 \mathrm{~s}$, que é tempo que a informação é atualizada no sistema de Automação. Essa taxa de atualização garante a vida útil da bateria de 2,9 anos conforme mostra a figura 4 abaixo. 


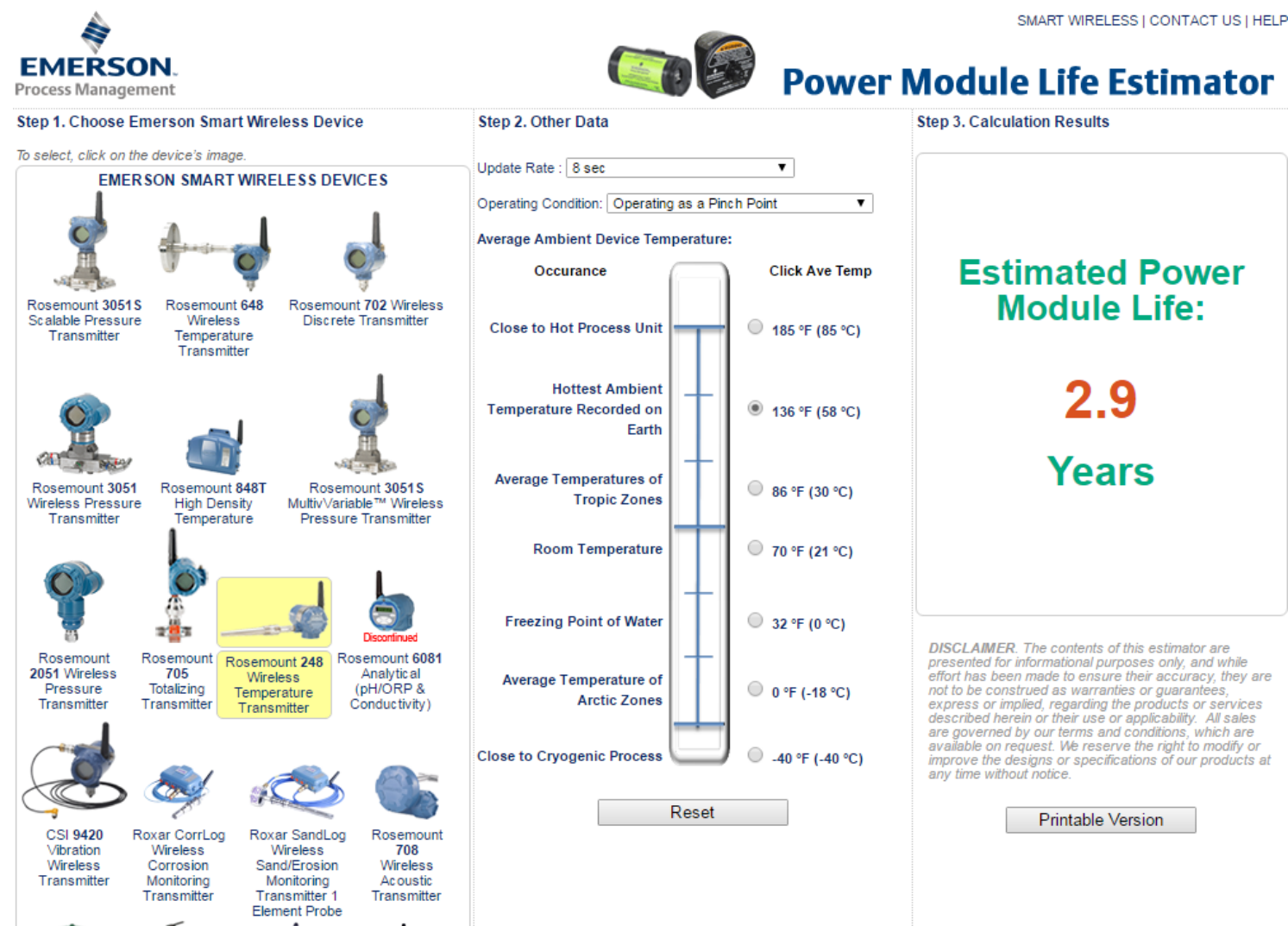

Figura 4 - 'Estimativa de vida útil bateria do Instrumento Wireless

\subsubsection{Projeto}

O projeto consistiu em integrar o sistema wireless da Emerson no sistema de automação PCS7 e uma rede wireless com alta disponibilidade e qualidade de transmissão. Para isso foi utilizado um gateway WirelessHART com comunicação modbus TCP/IP que é convertido para um protocolo de comunicação Profibus-DP através de um conversor da Prosoft. Na figura 5 mostra a arquitetura que foi desenvolvida.

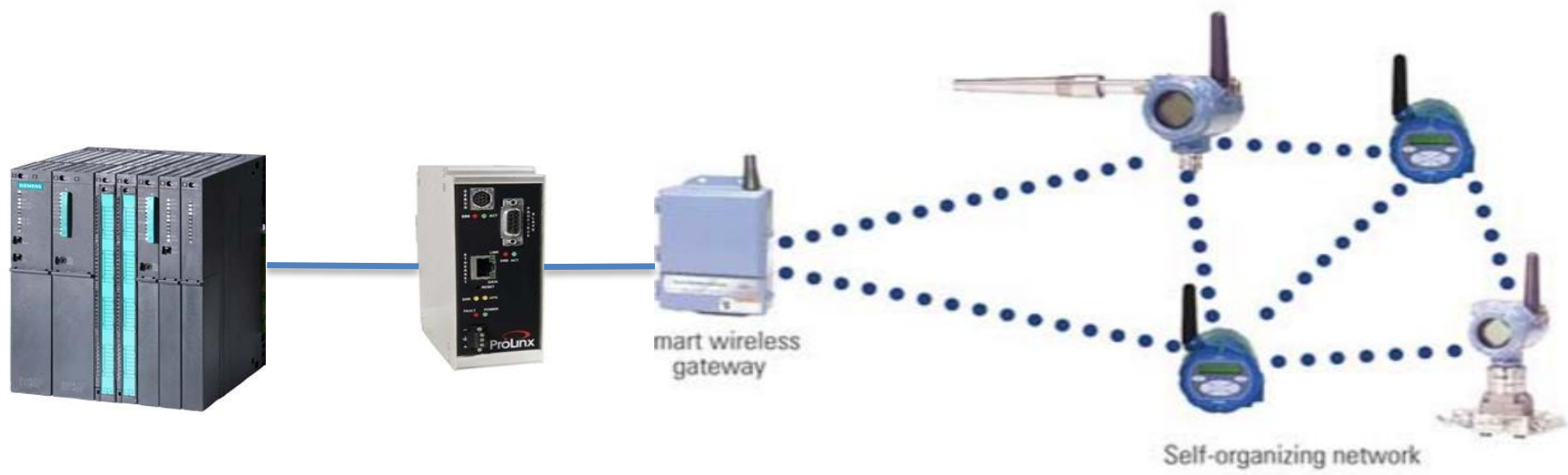

Figura 5 - Arquitetura da rede Wireless 
Na figura 6, mostra o painel onde alocam-se os componentes necessários para a arquitetura da rede WirelessHart da Emerson e a estrutura necessária para que a rede se comunique com o sistema de Automação Siemens PCS7.

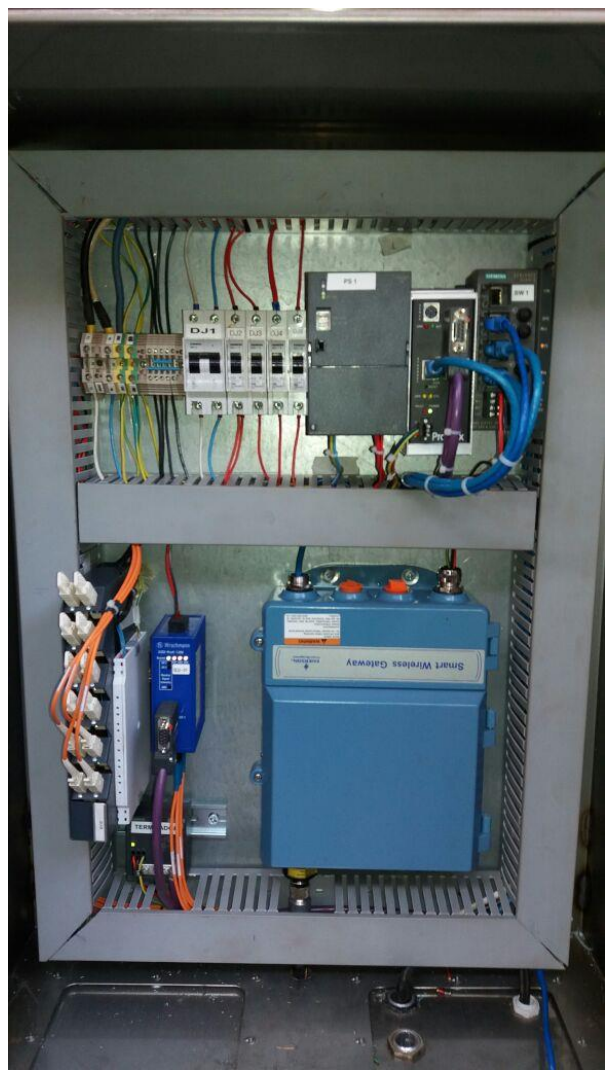

Figura 6 - Painel instalado do sistema wireless

Nesse projeto possibilitou o envio de 48 variáveis, sendo que atualmente são utilizadas 24 variáveis para as PV (variáveis de processo) e outras 24 para as variáveis que monitoram o nível de bateria. Caso o nível de bateria esteja baixo um alarme será acionado no sistema supervisório indicando sua troca. $\mathrm{Na}$ figura 7 indica a alocação dos Instrumentos.

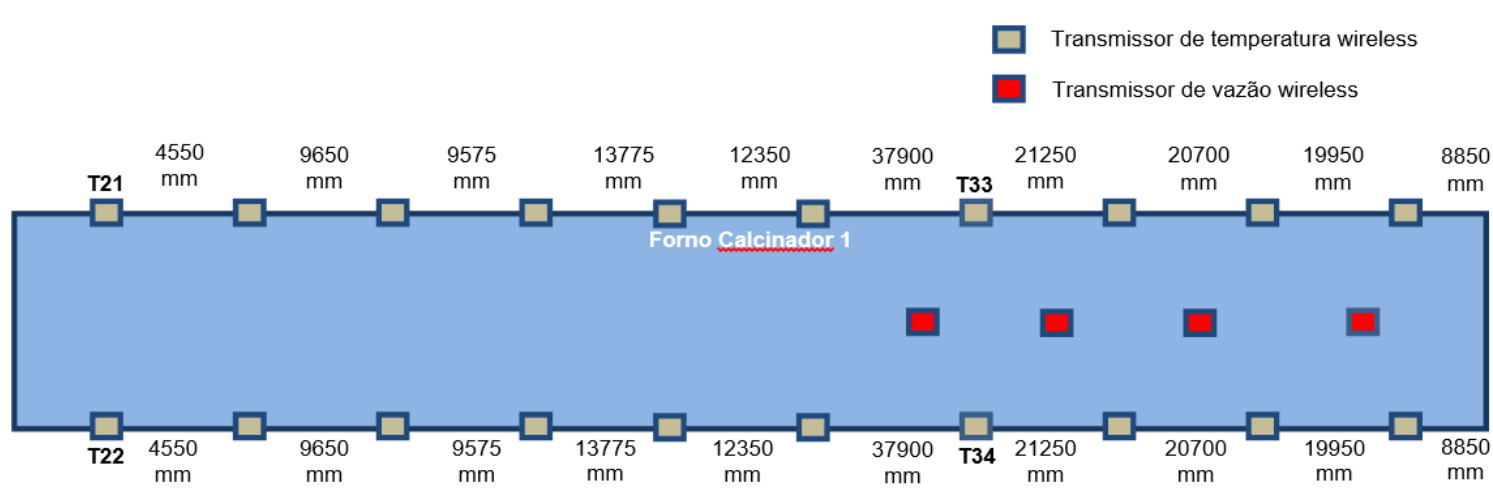

Figura 7 - Desenho esquemático com a instalação dos Instrumentos no forno 
A figura 8 mostra a comunicação entre um instrumento wireless e o gateway.

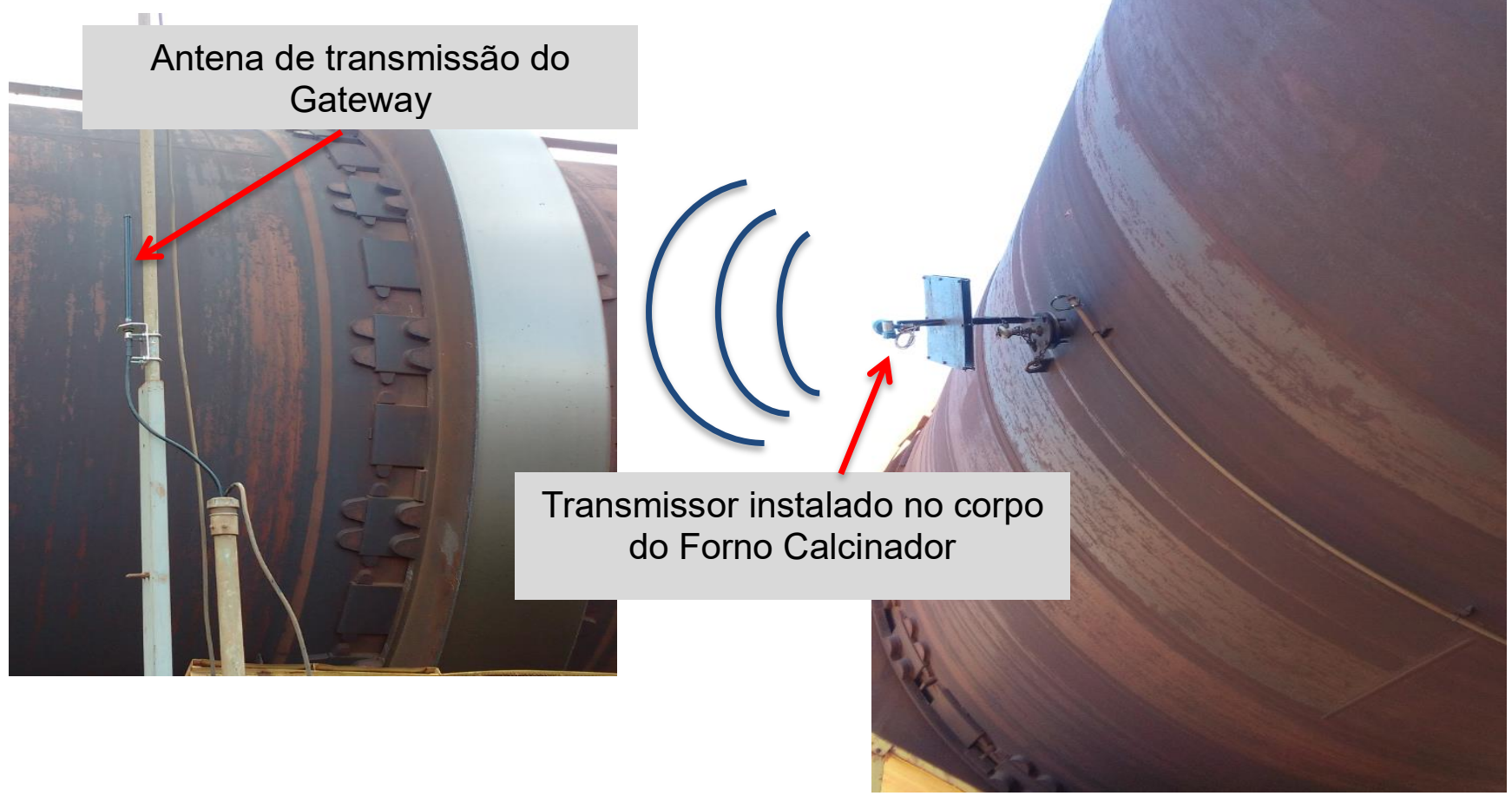

Figura 8 - Link de comunicação do Instrumento Wireless com o Gateway da rede

Um desafio para esse projeto foi a alta temperatura da carcaça do Forno Calcinador. Essa temperatura não poderia ser transmitida nem por radiação ou condução para a eletrônica do Transmissor wireless. Para solucionar o problema foi desenvolvido um suporte com um painel para isolamento térmico. Na figura 9 mostra o suporte desenvolvido.

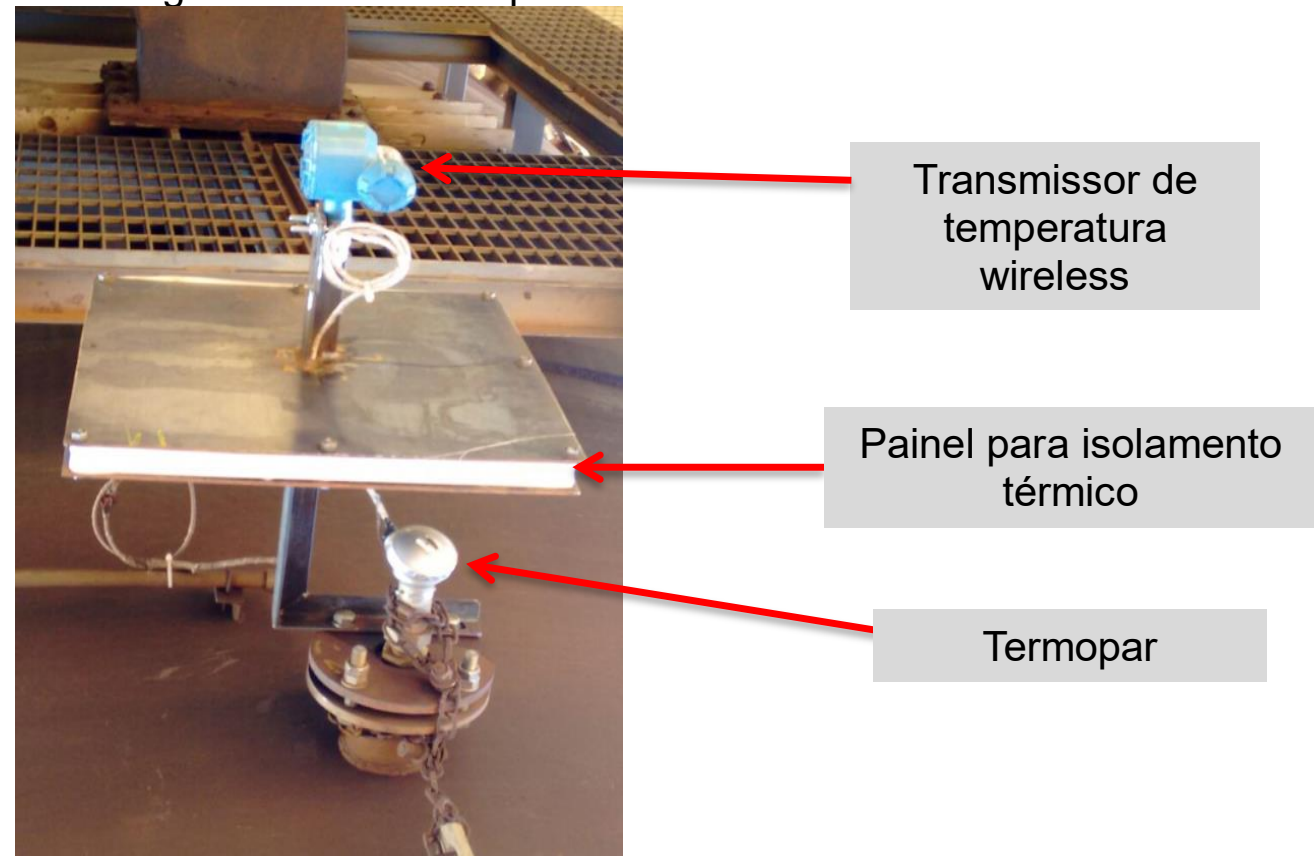

Figura 9 - Exemplo do suporte montado para proteger o Instrumento da alta temperatura do forno 


\section{RESULTADOS}

Os resultados alcançados foram extremamente positivos, o sistema wireless se comportou muito bem, o que permitiu a retirada completa do sistema original. Abaixo lista os resultados alcançados:

- O sistema se mostrou estável e não apresentou nenhuma perda de dados durante a operação do Forno Calcinador;

- A temperatura de trabalho do transmissor wireless ficou mais de $20^{\circ} \mathrm{C}$ abaixo do limite superior permitido na pior situação encontrada, mostrando que o isolamento térmico foi efetivo;

- Facilidade na manutenção, pois a única manutenção requerida após alguns anos de funcionamento é a troca da bateria do transmissor. Em caso de falha o MTTR será baixo porque esse sistema elimina cabos de transmissão, alimentação e um PLC's periféricos.

A figura 10 mostra a tela de um dos transmissores no qual apresenta a variável do processo, qualidade da rede, temperatura de eletrônica e nível de tensão da bateria.

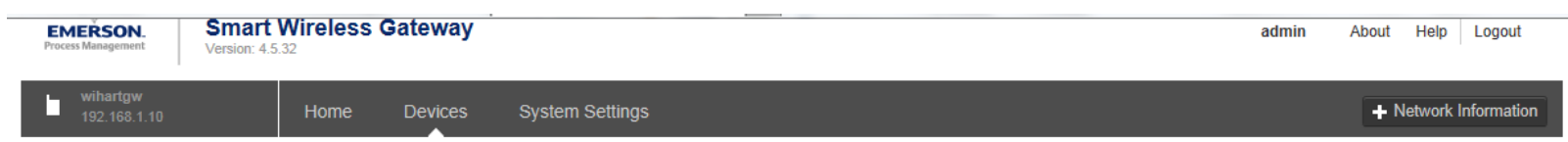
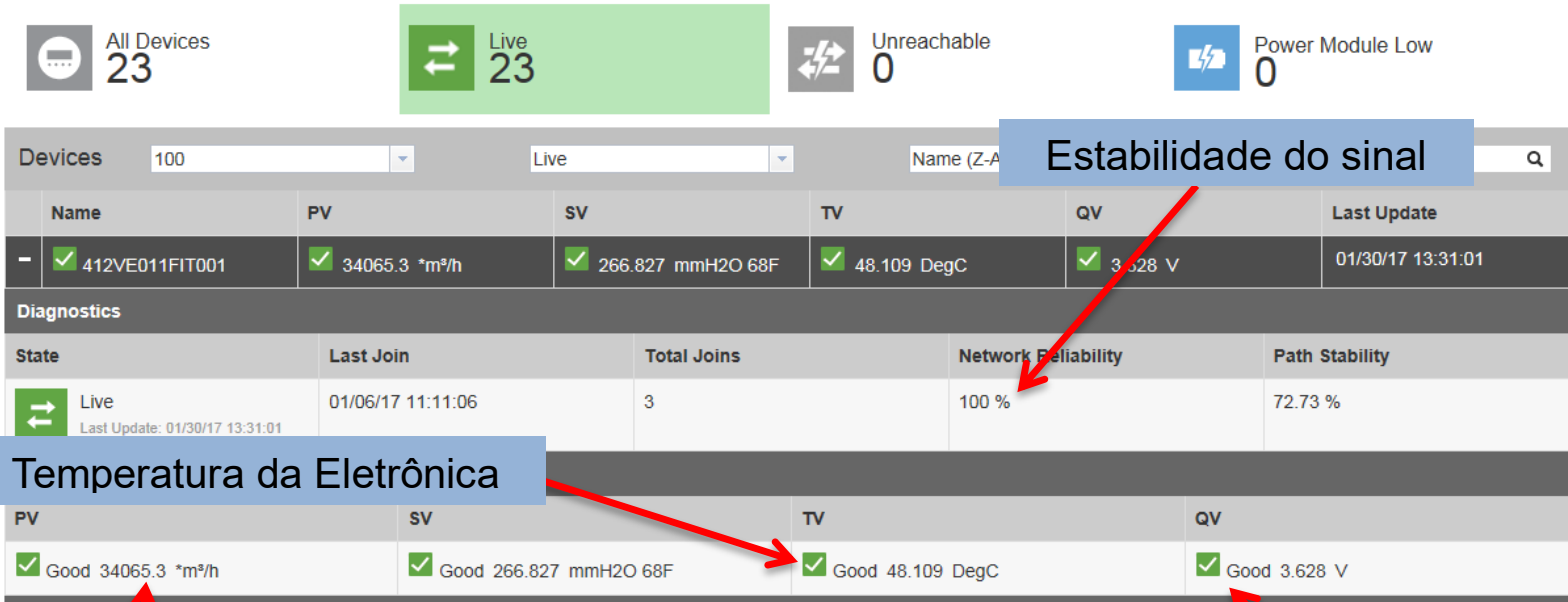

PV - Variável de processo

$$
\begin{aligned}
& \checkmark{ }_{30488.174}{ }^{*} \mathrm{~m}^{3 / h} \quad \nabla_{213.731 \mathrm{mmH} 2 \mathrm{O} 68 \mathrm{~F}} \quad \nabla_{49.229 \mathrm{DegC}}
\end{aligned}
$$

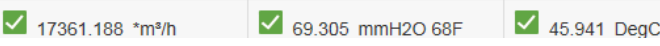

+ Detailed De nce Information

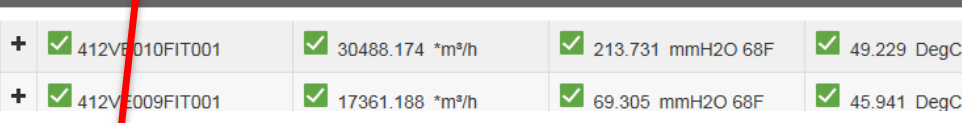

Figura 10 - Tela de status do Instrumento

Após esse projeto fica consolidada a tecnologia WirelessHart, que apresentou uma ótima performance e em nenhum momento apresentou perda de dados, ruídos na rede e interferência de outras frequências. 


\section{REFERÊNCIAS}

1 Reference Manual - WirelessHart Emerson 0089-0200-4420, Ver GA - Abril 2013;

2 Nixon, Mark - WirelessHart . 1aㅡ Edição Nova York: Springer Verglag

3 Smart Wireless da Emerson. Disponível em:

http://www2.emersonprocess.com/pt-

br/plantweb/wireless/Pages/WirelessHomePage-Flash.aspx Acesso em:

10/07/2016;

$4 \quad$ Smart Wireless da Emerson. Disponível em:

http://www2.emersonprocess.com/pt-br/brands/rosemount/temperature/singlepoint-measurement/248-wireless/pages/index.aspx Acesso em: 30/07/2016;

5 Smart Wireless da Emerson. Disponível em:

http://www2.emersonprocess.com/pt-br/brands/rosemount/pressure/pressuretransmitters/3051-pressure-transmitters/pages/index.aspx Acesso em: 30/07/2016; 